\title{
Comparison of Radiation Dose and Image Quality with Various Computed-Tomography Scout Views: The Angular Modulation Technique Based on Information Calculated from Scout Views
}

\author{
Myeong Seong Kim, ${ }^{1,2}$ Jong-Woong Lee, ${ }^{3}$ Sun Geun Kim, ${ }^{4}$ and Dae Cheol Kweon ${ }^{5,}$ \\ ${ }^{1}$ Department of Radiology, The Korean National Cancer Center, Goyang, Republic of Korea \\ ${ }^{2}$ Graduate School of Public Health and Institute of Health and Environment, Seoul National University, Seoul, Republic of Korea \\ ${ }^{3}$ Department of Radiology, Kyung Hee University Hospital at Gang-Dong, Seoul, Republic of Korea \\ ${ }^{4}$ Department of Radiological Technology, Baekseok Culture University, Cheonan, Republic of Korea \\ ${ }^{5}$ Department of Radiological Science, Shinhan University, Uijeongbu, Republic of Korea \\ "Corresponding author: Dae Cheol Kweon, Department of Radiologic Science, Shinhan University, Uijeongbu-shi, Republic of Korea. Tel: +82-318703416, Fax: +82-318703419, \\ E-mail: dckweon@gmail.com
}

Received 2015 December 19; Revised 2016 August 12; Accepted 2016 August 19.

\begin{abstract}
Background: While using the automatic exposure control technique in computed tomography(CT), the scout view determines the radiation dose for the $\mathrm{CT}$ scan by taking anatomical morphology into consideration.

Objectives: The goal of this study was to estimate radiation doses during scout scanning and to evaluate the hypothesis that the radiation dose during CT depends on the location and order of the X-ray tubes.

Materials and Methods: A CT scanner (GE VCT 64-channel) with the X-ray tubes placed in the anteroposterior (AP), lateral, and posteroanterior (PA) positions was used to acquire the scout views. The effective doses were assessed using an anthropomorphic chest phantom (RSD Inc., Long Beach, CA), an AAPM CT performance phantom (CIRS model 610), and CT dose indices (CTDI) head phantom. Both single and two scout views were tested with the three views combined according to the order of scanning. The CTDIs obtained from the CT unit dose report were used to compare the chest CT radiation doses, while the image quality was evaluated based on the root-mean-square error values calculated using the Image program.

Results: The results of this study indicate that single $\mathrm{AP}\left(0^{\circ}\right)$ scout views obtained with the z-axis modulation technique (AutomA) during chest CT scanning resulted in CTDIs that were lower than those generated by using lateral $\left(90^{\circ}\right)$ or PA $\left(180^{\circ}\right)$ scout views. The analysis of two-scout views using AutomA alone revealed the lowest CTDI in the $90^{\circ}-0^{\circ}$ scout views. However, based on the CT image noise analysis, a scout angle order of $0^{\circ}-90^{\circ}$ resulted in the optimal scout view combination with the minimal dose.

Conclusion: Scout views may be useful in determining dose and image quality in CT scanning; however, it is necessary to first determine the most appropriate scout view for CT scans.
\end{abstract}

Keywords: Automatic Exposure Control, Computed Tomography, Radiation Dose, Scout View

\section{Background}

The number of CT scans performed in the United States has continually increased in the recent years from 46 million in 2002 to 62 million in 2006 owing to the use of computed tomography (CT) imaging in hospital settings $(1,2)$. However, concerns have arisen regarding medical radiation exposure, and report that $1.5 \%$ to $2 \%$ of recent cancer occurrences in the United States may have been caused by CT scans (1-4). Consequently, numerous measures have been suggested to reduce the radiation doses from CT scans. However, reducing radiation levels without lowering image quality remains a challenge.

Among the proposed dose reduction methods, the automatic exposure control (AEC) technique can be used to perform CT studies without distortions, such as substantial increases in image noise and artifacts (3-6). Using this technique, scout scanning applies relatively lower and higher radiation doses for CT scanning within areas with lower and higher X-ray attenuation rates, respectively (59). In other words, AEC adapts the current tube for CT scanning in real time to account for the patient's body type, including body size, shape, anatomy, and the presence of prosthetic devices $(5,7-10)$.

By controlling radiation doses according to human body anatomy, the AEC technique relies on combining angular modulation along the $\mathrm{x}$ - and $\mathrm{y}$-axes (SmartmA; Figure $1 \mathrm{~A}$ ) and longitudinal modulation along the z-axis (AutomA; Figure $1 B$ ) (7). The desired level of image quality is determined by the noise index (reference dose: concept known as the desired level of image quality; GE Healthcare, Milwaukee, WI, U.S.A.) (8). A previous study showed that a normal human body is thicker laterally than frontally in a 
chest $\mathrm{CT}$, causing the lateral reduction to be more significant than the anteroposterior (AP) reduction (11). Consequently, in CT involving the AEC technique, the radiation dose in scout scanning is determined by the patient's body type, including body size, body shape, and anatomic location, as well as by the fixed noise index (9-12). As the scout information determines the radiation dose for a CT scan in the AEC technique, we speculated that the location and order of the X-ray tubes could also affect the radiation dose during a CT scan (13).

\section{Objectives}

The aim of the current study is to make a comparative assessment of the radiation dose and image quality in a helical CT scan after scout scanning, according to the location (angle) and order of the X-ray tubes.

\section{Materials and Methods}

\subsection{Multiple-Detector CT Scanning}

An anthropomorphic chest phantom (RSD Inc., Long Beach, CA, U.S.A.) was used for evaluation (Figure 2A) and was positioned supine and feet-first in a GE VCT 64-channel CT scanner (GE Healthcare, Milwaukee, WI, U.S.A.). For scout-center (axis) recognition, the sagittal and coronal planes of the phantom were set to the midline to reduce the dose effects caused by the location of the $\mathrm{x}$ - and $\mathrm{y}$ axes. A standard 16-cm-diameter computed tomography dose index (CTDI) head phantom was used for varied quantification of the radiation dose based on the location $\left(0^{\circ}\right.$, $90^{\circ}, 180^{\circ}$, and center) (Figure $2 \mathrm{~B}$ ). The CTDI head phantom was made of polymethylmethacrylate (i.e., acrylic, Lucite) and contained five holes to accept pencil-shaped ionization chambers. One of the holes was in the center, and the others were located at $0^{\circ}, 90^{\circ}, 180^{\circ}$, and $270^{\circ}$, each at a distance of $1 \mathrm{~cm}$ from the surface of the phantom (Figures 2C and 2D). An American Association of Physicists in Medicine (AAPM) CT performance phantom (CIRS model 610) was used to evaluate the impact of the CT tube angle on the radiation dose with different chest phantom morphologies (Figure 2E).

When a CT study is performed with AEC, radiation exposure during the helical CT scan is automatically controlled based on the density (size and attenuation) of the body part being scanned in order to achieve identical image quality with lower radiation doses. Thus, in this study, anthropomorphic chest and AAPM phantom were used to know influence of variations in the radiation doses and the image quality in helical CT scan from a different phantom characteristic (a true with identical density and an ovoid with different attenuations of body parts).

Every scout scan was performed using a tube voltage and tube current of $120 \mathrm{kVp}$ and $10 \mathrm{~mA}$, respectively. After scout scanning, the chest CT scanning employed a $36 \mathrm{~cm}$ display field of view with the apex set to the lower level of the chest scan length $(30 \mathrm{~cm})$. Each scout-view scan was followed by chest CT under the same conditions (Table 1). The same conditions were also applied to the AAPM phantom.

Table 1. Chest CT Study Parameters

\begin{tabular}{|lc}
\hline Parameter & Value \\
\hline Tube voltage of scout (kVp) & 120 \\
\hline Tube current of scout (mA) & 10 \\
\hline Noise index & 18 \\
\hline Scan type & Helical \\
\hline Rotation time (s) & 0.5 \\
\hline Rotation length & Full \\
\hline Interval (mm) & 10.0 \\
\hline SFOV & Large body \\
\hline Helical thickness (mm) & 5.0 \\
\hline Detector coverage & 40.0 \\
\hline Pitch and speed (mm. rot) & 30 \\
\hline DFOV (cm) & $0.984: 139.37$ \\
\hline Scan length (cm) & 36 \\
\hline
\end{tabular}

Abbreviations: SFOV, Scan field of view; DFOV, Display field of view.

To minimize the deviation, determination of the amount of radiation doses were repeated three times in similar ways. The resulting scanned radiation doses were averaged in this study.

A calibrated ionization chamber (UNFORS Xi, Raysafe AB, Billda, Sweden) was used to measure the radiation dose at each position of the CTDI head phantom. Before starting the experiment, the ionization chamber was placed in the CT room for an hour under constant temperature and humidity $\left(21^{\circ} \mathrm{C}\right.$ and $\left.45 \%\right)$, to enable more accurate estimation of the radiation dose. The CTDI head phantom CT scanning 
A

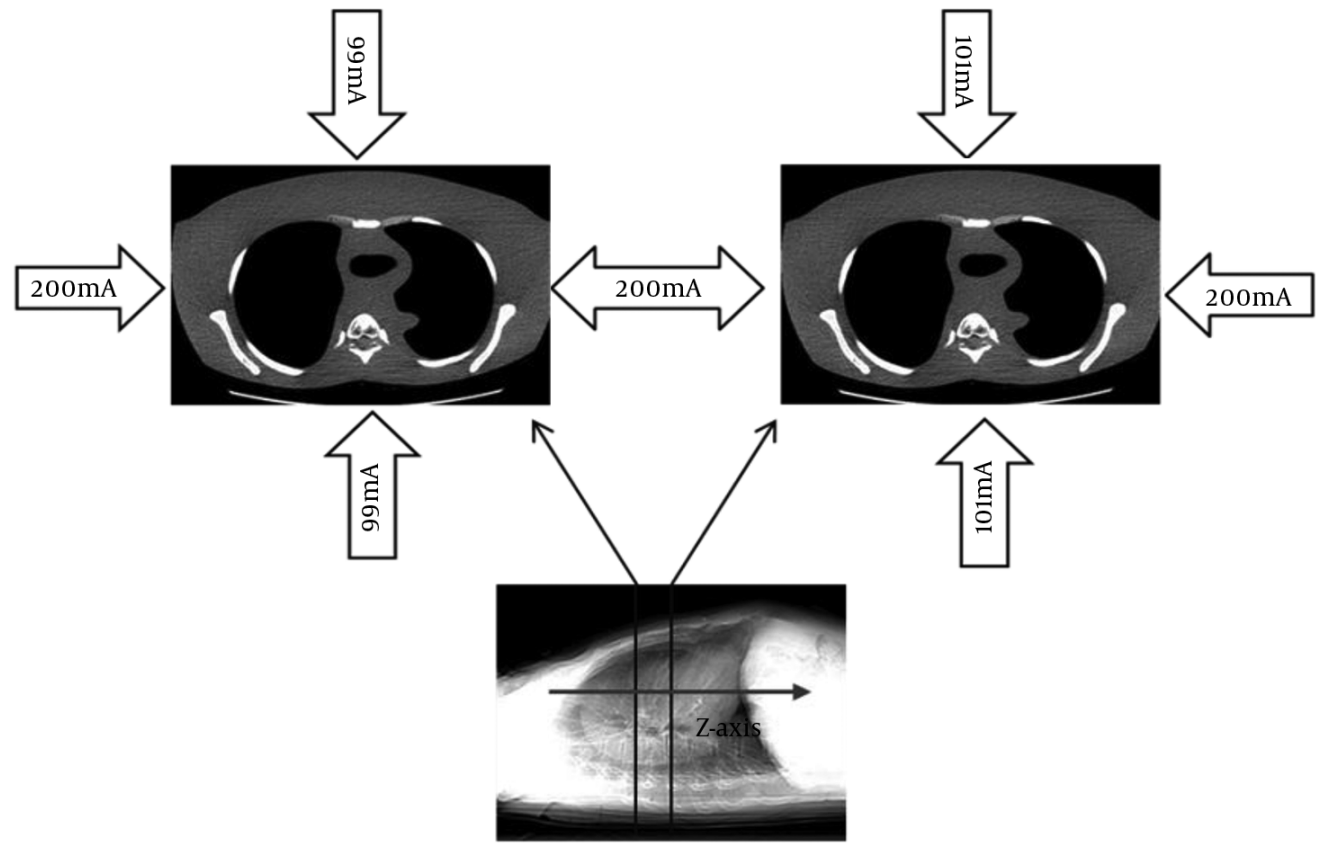

B

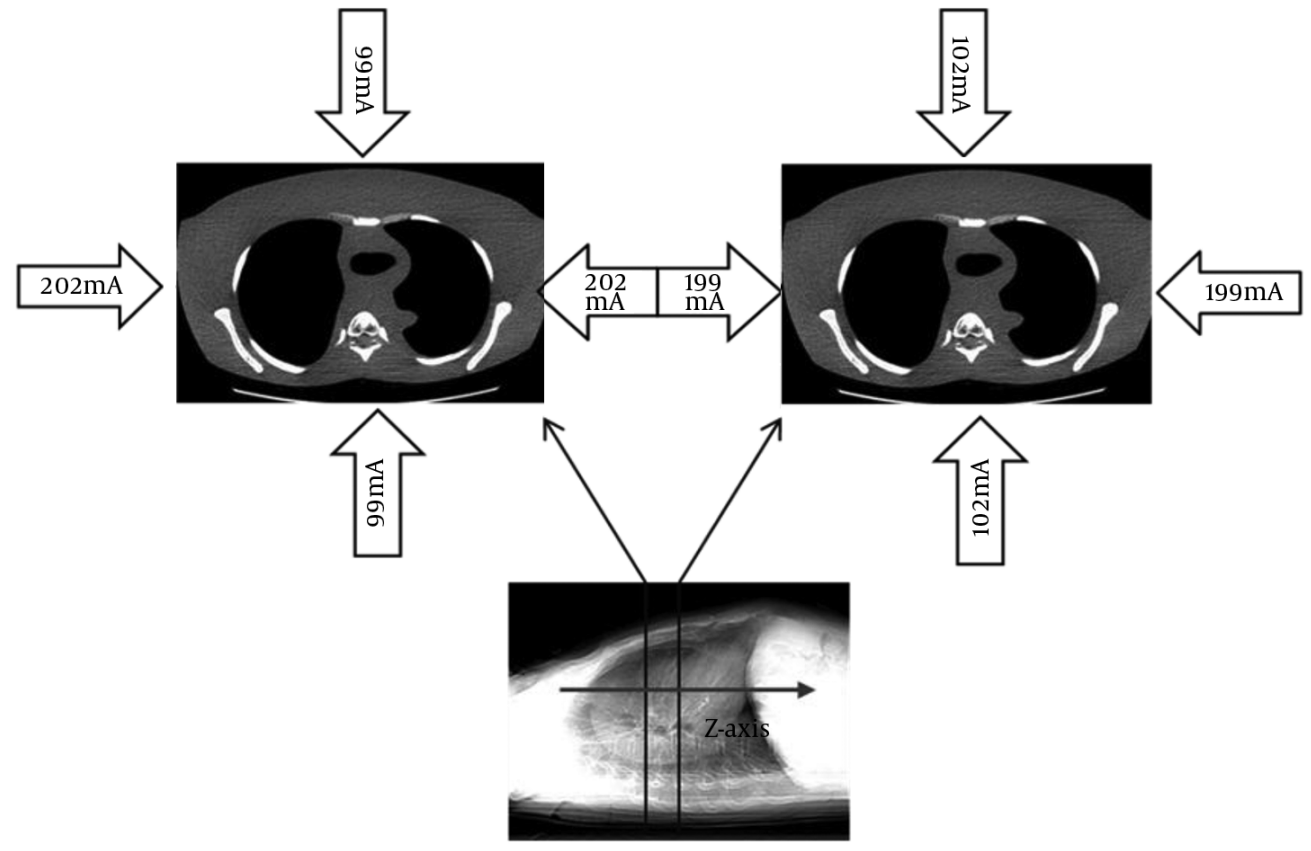

Figure 1. Automatic exposure control (AEC) technique; A, SmartmA; B, AutomA. 

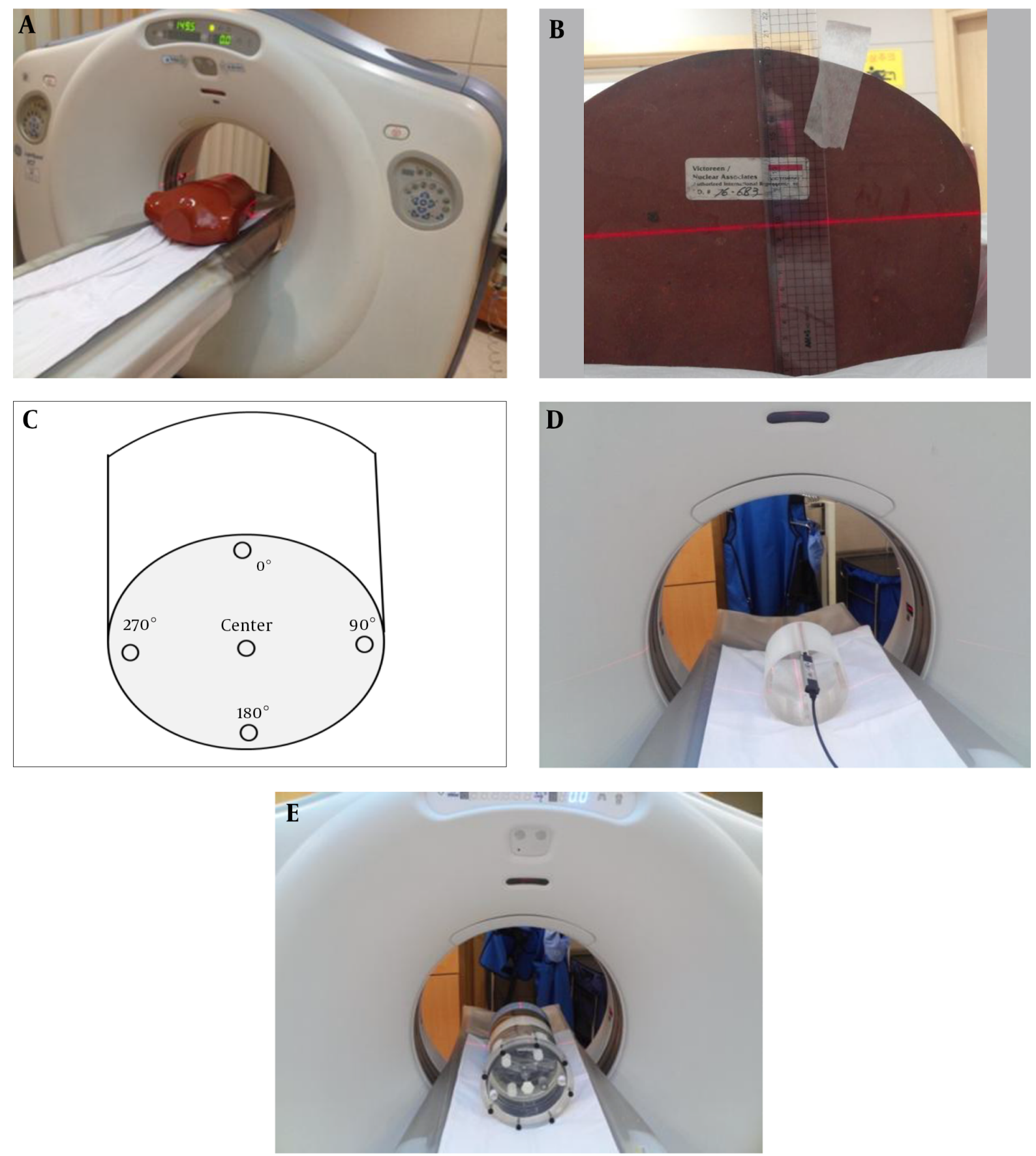

Figure 2. Using varied phantoms for the scout center in the GE VCT 64-channel CT scanner; A, An anthropomorphic chest phantom; B, Scout center (laser beam) is placed at the center of the y-axis on an anthropomorphic chest phantom; C, A depiction of the placement of holes in the computed tomography dose index (CTDI) head phantom; D, A CTDI head phantom; E, An AAPM phantom. 
conditions were the same as those described in Table 1, except that the tube current was changed from $10 \mathrm{~mA}$ to 50 $\mathrm{mA}$.

\subsection{Scanning Protocols}

In this study, the X-ray tube placements for the basic scout views were anteroposterior (AP), lateral, and posteroanterior (PA), as the effects of the table and variations in the distance between the X-ray tubes and detector were suspected to have significant effects on the dose (13). To assess the effects of using different scout views on the helical CT scan (chest CT scan with anthropomorphic chest phantom), the scout views were divided into single scout views of each of the three basic types $\left(0^{\circ}, 90^{\circ}\right.$, and $\left.180^{\circ}\right)$ and combinations of two-scout views in different directions $\left(0^{\circ}, 90^{\circ}\right.$, and $\left.180^{\circ}\right)$. In other words, the single-scout views had the X-ray tube acquire $0^{\circ}, 90^{\circ}$, or $180^{\circ}$ scout views before chest CT scanning (Figure 3A), while the two-scout views used combinations of two of the basic $0^{\circ}, 90^{\circ}$, and $180^{\circ}$ scout views in different directions, according to the order of scanning: $0^{\circ}-90^{\circ}, 90^{\circ}-0^{\circ}, 90^{\circ}-180^{\circ}$, and $180^{\circ}-90^{\circ}$ (Figure $3 \mathrm{~B}$ ). As the two-scout view applications could employ SmartmA, tests were conducted with AutomA alone as well as with both AutomA and SmartmA.

\subsection{Evaluation of Image Quality}

An AW4.0 workstation (Advantage Windows; GE Healthcare, Milwaukee, WI, U.S.A.) was used for the image analysis. For comparison with the chest CT radiation dose while using the anthropomorphic chest phantom and the AAPM, the CTDI volume (CTDIvol; mGy) and the dose-length product (DLP; $\mathrm{mGy} . \mathrm{cm}$ ) were used; the results from CT scanning were displayed on the dose report of the CT scanner. The root-mean-square error (RMSE) scores of the helical scans of the anthropomorphic chest phantom were obtained from the Image software (version 1.48e; NIH Image; free download available at http://rsb.info.nih.gov/ij/download.html) and were used for quantitative comparison of the qualities of the chest CT images obtained from the different scout views. An Image signal-to-noise ratio plug-in was used to obtain RMSE estimates for comparison, using a $0^{\circ}$ scout view (Figure $3 \mathrm{~A})$ as a reference and test image. The following ratio was used for an assessment of the quantitative radiation dose and for relative comparison of the image quality:

Ratio $=$ RMSE $/(10-$ CTDIvol (mGy)).

The dose variations resulting from different scout views were compared by estimating the rates of radiation (Table 2 ) according to the ratio.

Radiation increase ratio $=($ CTDI value of test scout angle)/4.23 (CTDI value of helical scanned after $0^{\circ}$ scout view).
To evaluate the chest CT image quality in detail, we identified five regions of interest (ROIs) in the main sections (Figure 4A). ROI 1 represented the background; ROI 2, located in the chest wall, represented the anterior effects; ROI 3, located in the lung, was used to evaluate the lung field; ROI 4, located in the anterior spine in the middle of the chest phantom, was used to evaluate the effects on the posterior spine; and ROI 5, located in the back muscle, represented the posterior effects. Each of these ROIs was assessed for noise.

The ImPACT CT patient dosimetry calculator (version 1.0.2, St George's Healthcare NHS Trust, Tooting, England) was used to estimate the effective dose. Recommendations from Publication 103 of the International Commission on Radiological Protection were used to calculate the effective doses in this study.

\subsection{Statistical Analysis}

We conducted paired t-tests (SPSS for Windows, version 7.5; SPSS Inc., Chicago, IL, U.S.A.) to determine whether AutomA or AutomA and SmartmA combined yielded significantly different CTDI values when two-scout views were used. Values of P less than 0.05 were considered statistically significant (differences: ${ }^{*} \mathrm{P}<0.05$ ).

\section{Results}

The relative dose variations in the CTDIvol values for the various scout views and the differences between the dose values obtained using the tested scout views and that obtained using the $0^{\circ}$ reference scout view (which was taken to be $0 \%$ ) are presented in Table 2 .

While the two-scout view applications did not cause constant variation in the CTDIvol, as seen in the single scout views, they did result in some characteristic changes. Among the combinations of lateral and frontal scout images, $90^{\circ}-180^{\circ}($ CTDIvol $=5.03 \mathrm{mGy}$ and RMSE $=117.5)$ yielded a similar RMSE score but a higher CTDI than that produced by $90^{\circ}-0^{\circ}(\mathrm{CTDIvol}=4.23 \mathrm{mGy}$ and $\mathrm{RMSE}=115.4)$. In contrast, among the combinations of frontal and lateral scout image views, $180^{\circ}-90^{\circ}(\mathrm{CTDIvol}=4.32 \mathrm{mGy}$ and $\mathrm{RMSE}$ $=104.9$ ) yielded the same CTDIvol but a higher RMSE score than $0^{\circ}-90^{\circ}$ produced $($ CTDIvol $=4.32 \mathrm{mGy}$ and $\mathrm{RMSE}=$ 75.7). The $90^{\circ}-0^{\circ}$ scout view had a $2.12 \%$ lower dose but up to a $32.17 \%$ higher RMSE score than the $0^{\circ}-90^{\circ}$ scout view, as well as a significant increase in noise. The $90^{\circ}$ $180^{\circ}$ scout view had a $19.14 \%$ higher dose and up to $64.4 \%$ and $25.26 \%$ higher RMSE scores than the $0^{\circ}$ reference and $0^{\circ}-90^{\circ}$ scout views, respectively. Ultimately, while the $90^{\circ}$ $-0^{\circ}$ scout view had the lowest dose, the single $180^{\circ}$ and $0^{\circ}$. $90^{\circ}$ two-scout views had both the smallest RMSE scores and dose-to-image-noise ratios (Table 2). 

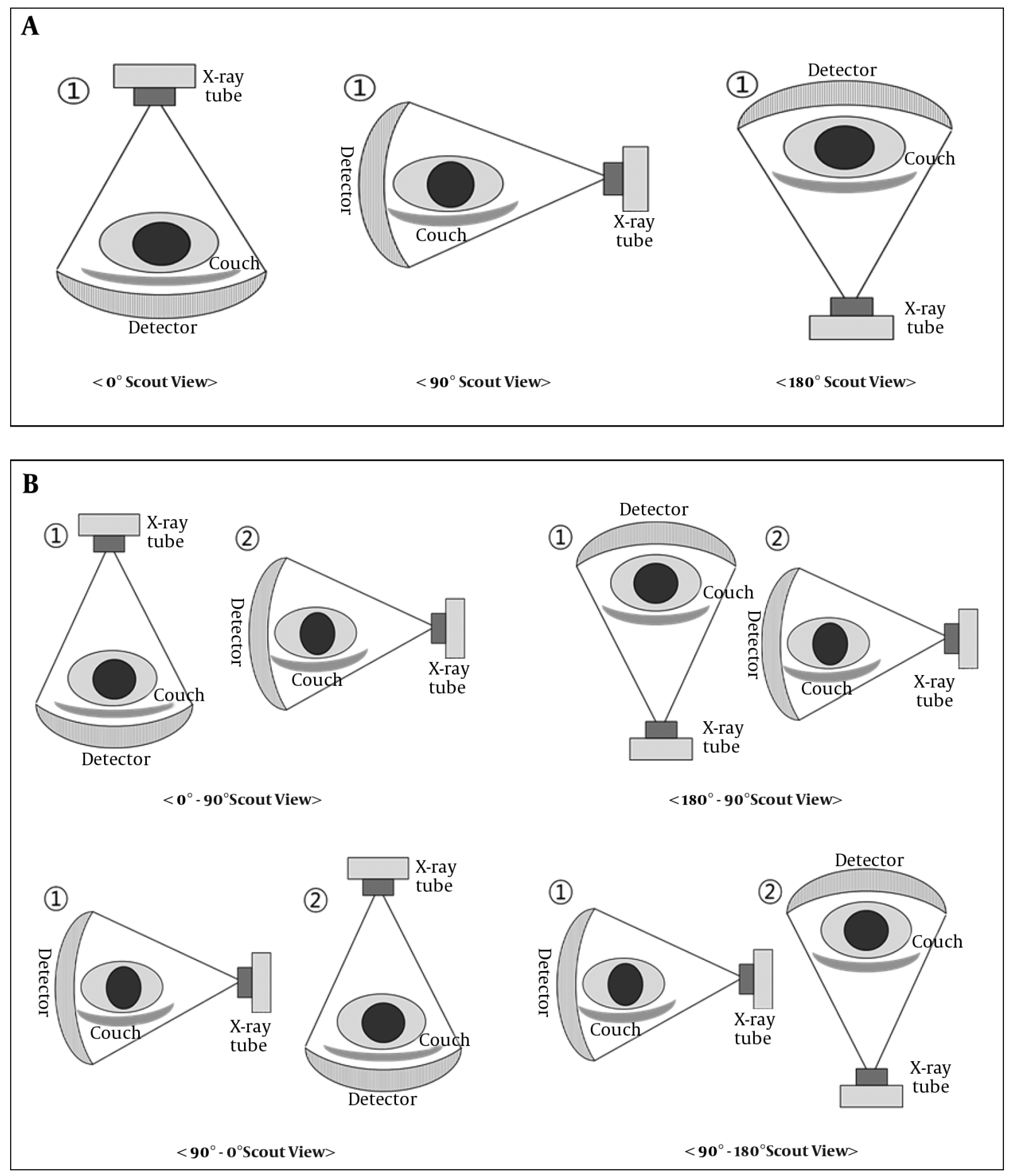

Figure 3. Application of scout view on the anthropomorphic chest phantom; A, The single scout view application test; B, The two scout view application test.

Analysis of the single scout view results showed that $180^{\circ}(\mathrm{CTDI}=5.04 \mathrm{mGy}$ and $\mathrm{RMSE}=41.9)$ resulted in the highest chest CTDI and lowest RMSE score (Table 2, Figure
$5 \mathrm{~A})$, followed by $90^{\circ}(\mathrm{CTDI}=4.32 \mathrm{mGy}$ and $\mathrm{RMSE}=71.8)$ and $0^{\circ}$ (CTDI $\left.=4.23 \mathrm{mGy}\right)$. Thus, with the $0^{\circ}$ scout view as the reference, a normal pattern of dose increase and noise 
Table 2. Dose Variation, CTDIvol, Effective Dose, RMSE, and Ratio According to the Application of Scout View Using Anthropomorphic Chest Phantom

\begin{tabular}{|c|c|c|c|c|c|c|c|}
\hline \multirow[b]{2}{*}{ Scout View } & \multicolumn{3}{|c|}{ AutomA (Single scout view) } & \multicolumn{4}{|c|}{ AutomA/AutomA with SmartmA (Two scout view) } \\
\hline & $0^{\circ}$ & $90^{\circ}$ & $180^{\circ}$ & $0^{\circ}-90^{\circ}$ & $180^{\circ}-90^{\circ}$ & $90^{\circ}-0^{\circ}$ & $90^{\circ}-180^{\circ}$ \\
\hline \multicolumn{8}{|l|}{ Average (value) } \\
\hline CTDIvol (mGy) mean \pm SD & $4.23 \pm 0.32$ & $4.32 \pm 0.01$ & $5.04 \pm 0.28$ & $4.32 / 4.25$ & $4.32 / 4.25$ & $4.23 / 4.21$ & $5.04 / 4.61$ \\
\hline $\mathrm{P}^{\mathrm{a}}$ & \multicolumn{3}{|c|}{0.89} & \multicolumn{4}{|c|}{0.99} \\
\hline $\begin{array}{l}\text { Radiation increase rate (Reference: single-scout } \\
\text { view } 0^{\circ}(0 \%) \text { ) }\end{array}$ & Reference ( $0 \%)$ & $2.12 \%$ & $19.14 \%$ & $2.12 \% / 0.47 \%$ & $2.12 \% / 0.47 \%$ & $0 \mid-0.48 \%$ & $19.14 \%$ / 8.98\% \\
\hline Effective Dose (mSv) & 2.63 & 2.69 & 3.14 & $2.69 / 2.65$ & $2.69 / 2.65$ & $2.63 / 2.62$ & $3.14 / 2.87$ \\
\hline RMSE & Reference $(0)$ & 71.8 & 41.9 & $75.7 / 76.7$ & $104.9 / 105.5$ & $115.4 / 115.9$ & $117.5 / 118$ \\
\hline Ratio RMSE/(10-CTDIvol) & Reference $(0)$ & 12.64 & 8.44 & $13.32 / 13.34$ & $18.46 / 18.34$ & $20 / 20.01$ & $23.64 / 21.89$ \\
\hline
\end{tabular}

Abbreviatins: CTDIvol, computed tomography dose index volume; RMSE, root-mean-square error; SD, standard deviation.

${ }^{\mathrm{a}}$ The Average value is the mean of three times measurements.

decrease is evident, with higher doses resulting in lower RMSE scores (Table 2). The $180^{\circ}$ scout view has a dose $19.1 \%$ higher than that obtained using the $0^{\circ}$ scout view (Table 2 ).

Using both AutomA and SmartmA resulted in a CTDI slightly lower than that obtained using only AutomA, but the difference was not statistically significant (Table 2). In contrast, using only AutomA resulted in RMSE scores slightly lower than those obtained using both AutomA and SmartmA (Figure 4B).

Image analysis at each of the ROIs in the phantom chest CT images revealed that the single $180^{\circ}$ scout view generally led to lower noise values but also to slightly higher relative noise values in the anterior area (ROI 2) and the anterior spine (ROI 4) (Figure 5B).

Among the two-scout views with $90^{\circ}$ combinations of the second scout views, the $0^{\circ}-90^{\circ}$ scout view had lower noise values in the background and back muscle areas (ROIs 1 and 5, respectively), and higher noise values in the other areas, compared to those of the $180^{\circ}-90^{\circ}$ scout view (Figure 5B). Regarding the two-scout views with the frontal images from the second scout views, the $90^{\circ}-0^{\circ}$ scout view had noise levels slightly higher than those of the $90^{\circ}-180^{\circ}$ scout view at ROIs other than the anterior spine and back muscle (ROIs 4 and 5, respectively (Figure 5B). The $90^{\circ}-180^{\circ}$ scout view had the lowest noise value at the lung field (ROI 3 ) on the chest CT images, which was an expected outcome given the dose (Figure $5 \mathrm{~B}$ ).

The noise analysis in the chest CT ROIs also indicated that using AutomA alone yielded similar results to those obtained from using both AutomA and SmartmA (Figure 5C).

The radiation doses at the various locations on the CTDI head phantom exhibited a pattern similar to that of the doses at the corresponding locations on the anthropomor- phic chest CT phantom (Figure 6). Specifically, the radiation dose at the $0^{\circ}$ location was higher than the dose at the $90^{\circ}$ location on the CTDI head phantom, and the dose at the $90^{\circ}$ location was higher than at the $180^{\circ}$ location. Additionally, the higher tube current (100 kVp vs $120 \mathrm{kVp}$ ) led to the more differences of radiation dose that depended on the location on the CTDI head phantom (Figure 6).

\section{Discussion}

The AEC technique uses information obtained from two-scout scans acquired in the AP and lateral views. Using this information, sinusoidal modulation of the tube current is achieved during $360^{\circ}$ rotation in order to equalize X-ray absorption $(9,13)$. Therefore, rotational or angular dose modulation involves varying the tube current to equalize the photon flux to the detector as the X-ray tube rotates about the patient (e.g., from the AP position to the lateral position). The radiation doses obtained using different scout views were verified using the CTDI head phantom. It was found that the attenuation rate in the CT system was greater at the $180^{\circ}$ position than it was at the $0^{\circ}$ position; the dose in the AEC helical scan was based on this result, so the $180^{\circ}$ scout view resulted in a dose higher than that yielded by the $0^{\circ}$ scout view.

Table 2 shows the CTDIvol and RMSE values based on the reference $0^{\circ}$ scout view and the RMSE/(10 - CTDIvol) ratios of the single scout views. The $180^{\circ}$ scout view has CTDIvol and RMSE scores that are higher and lower, respectively than those of the $90^{\circ}$ scout view. Additionally, the $180^{\circ}$ scout view (RMSE/(10 - CTDIvol) $\left.=8.44\right)$ has an RMSE/(10 - CTDIvol) ratio lower than that of the $90^{\circ}$ scout view $($ RMSE $/(10-$ CTDIvol $)=12.64 \%)$. Furthermore, the $180^{\circ}$ scout view yielded images of higher quality than the $90^{\circ}$ 
A

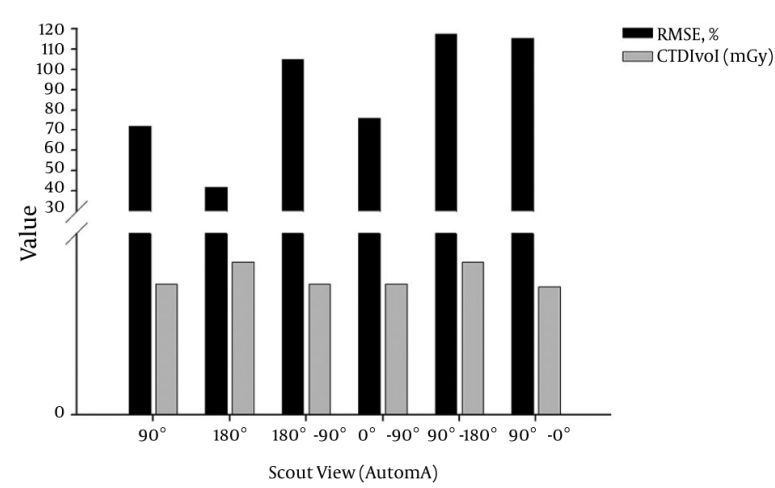

B

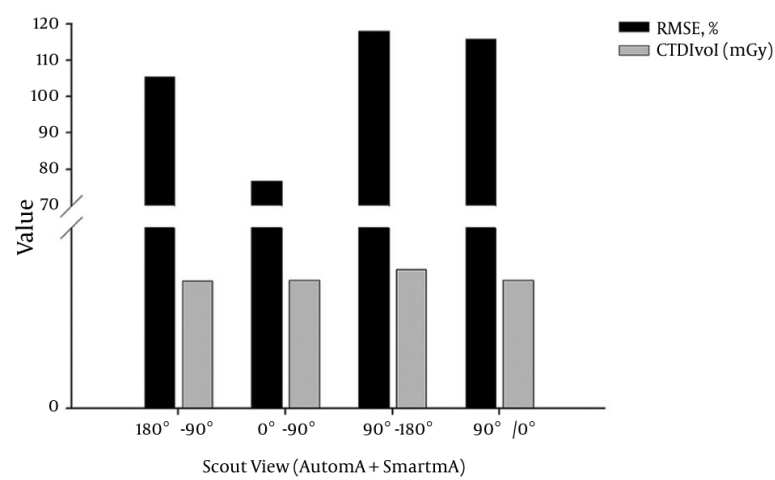

Figure 4. Comparison of root-mean-square error (RMSE) on chest phantom CT images; A, RMSE by scout views with the application of AutomA alone; B, RMSE by scout views with the application of both AutomA and SmartmA

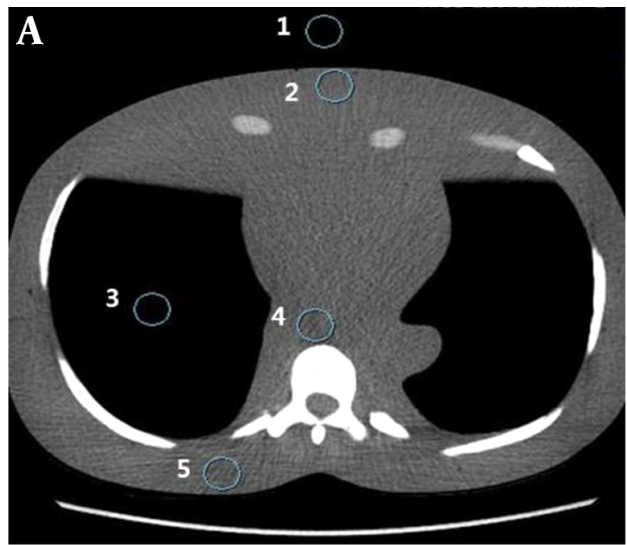

B

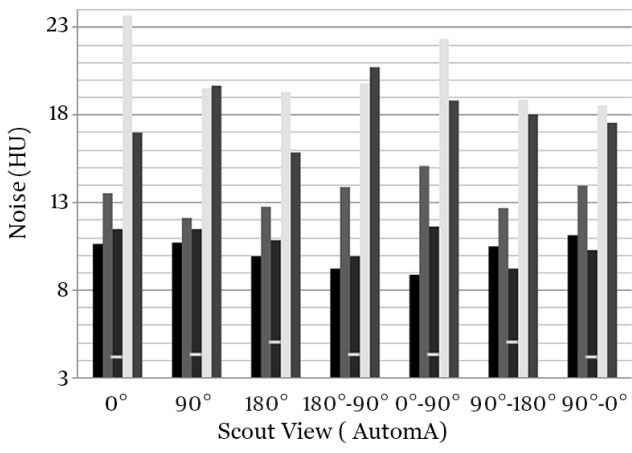

C

- (1) ROI

- (2) $\mathrm{ROI}$

- (3) ROI

(4) ROI

- (5) ROI

- CTDIvol

Figure 5. Comparison of noise at each area on the anthropomorphic chest phantom CT images; A, The region of interest (ROI) in the main sections on the chest phantom CT images; B, Noise by scout views with the application of AutomA alone; C, Noise by scout views with the application of both AutomA and SmartmA.

scout view did, owing to the increased dose and reduced noise of the former view. Generally, the $90^{\circ}$ scout views led to higher doses than the $0^{\circ}$ scout views, most likely be- cause lateral projections result in X-ray attenuation greater than that produced by projections $\left(0^{\circ}\right)$, particularly in the regions of the body, such as the shoulders and pelvis, that 
A

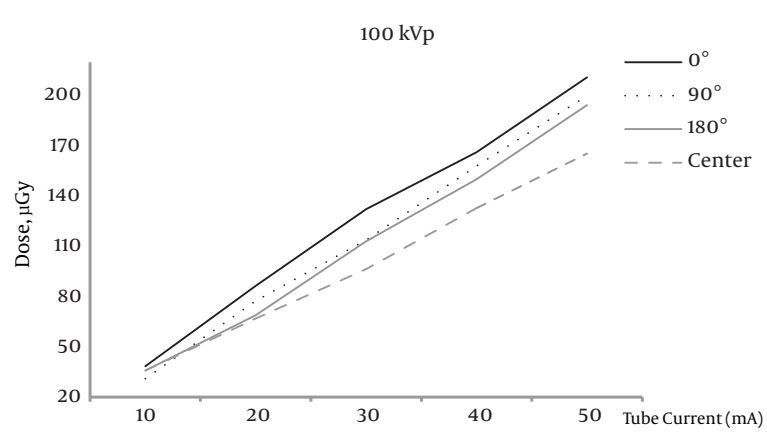

B

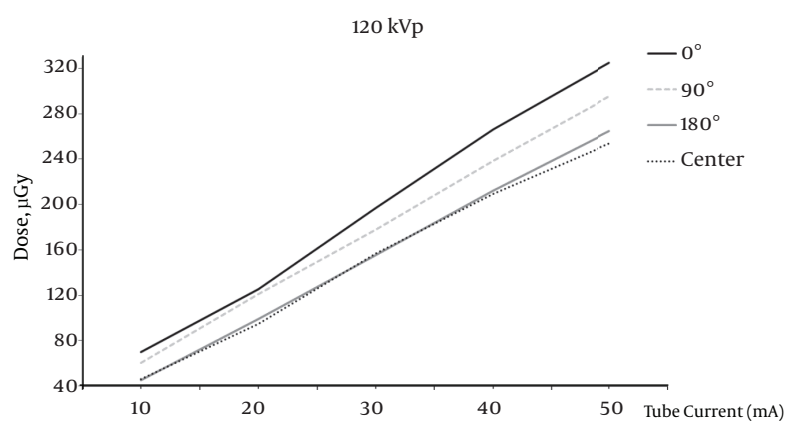

Figure 6. Comparison of radiation dose distribution according to the location (location hole at $0^{\circ}, 90^{\circ}, 180^{\circ}$, and the center) in the CT dose index (CTDI) head phantom; A, The radiation dose depends on variation in angular positioning of the CTDI head phantom using an ionization chamber dosimeter at 100kVp; B, The radiation dose depends on variation in the angular position of the CTDI head phantom using the ionization chamber dosimeter at 120kVp; The radiation doses at various locations on the CTDI head phantom exhibit a pattern similar to that of the doses at the corresponding locations on the chest CT phantom.

have more asymmetric oval shapes than does the head. The $180^{\circ}$ scout view yielded X-ray attenuation greater than that of the $0^{\circ}$ scout view, most likely due to the couch on which the patient was lying (14). The same CT-scanning conditions used for the chest phantom were applied to the AAPM phantom, with the same CTDI (CTDIvol = $4.32 \mathrm{mGy}$ ) values (Table 3). Consequently, no measurable dose differences between the scout views and the cylindrical object (phantom with circular type: AAPM phantom) were observed.

Table 3. Computed Tomography Dose Index Volume (CTDI vol) (mGy) Using the Scout View Application in the AAPM Phantom ${ }^{\mathrm{a}}$

\begin{tabular}{lccc}
\hline & \multicolumn{3}{c}{ AutomA } \\
\hline Scout View & $0^{\circ}$ & $90^{\circ}$ & $180^{\circ}$ \\
CTDIvol (mGy) & $4.32 \pm 0.01$ & $4.32 \pm 0.01$ & $4.32 \pm 0.01$ \\
P Repeated 3 Times & & 0.99 & \\
\hline
\end{tabular}

${ }^{\mathrm{a}}$ Values are means \pm standard deviations.

The scout-view-based angular modulation technique (SmartScan, GE Power, U.S.A.) provides a means for estimating tube current for different projection angles over $360^{\circ}$ rotations around the object being scanned (Table 2) (7). Thus, the technique modulates radiation doses based on information (shape and attenuation) obtained from a single scout view (latter scout view). In Table 2, the two-scout views revealed that the final dose depended, in each case, on the latter view. For instance, when the latter of the twoscout views was $90^{\circ}$, both the $0^{\circ}-90^{\circ}$ and $180^{\circ}-90^{\circ}$ scout views resulted in the same dose. Therefore, the latter scout view determined the dose.

Our results indicate that higher CTDIs in single scout view applications resulted in smaller corresponding noise values in all five of the ROIs (Figure 5B). In contrast, the twoscout views tended to exhibit different noise values in the five ROIs, which depended on the scout view rather than the CTDI. Despite having equivalent CTDI values, the combinations of $180^{\circ}-90^{\circ}$ and $0^{\circ}-90^{\circ}$ scout views, which represented APviews, resulted in different noise values in ROIs 2 and 5, respectively. These results are likely due to the distance between the entrance and exit points of the X-rays on the anthropomorphic chest phantom, which could cause variation in the dose reduction and might lead to dose differences between the front and back sides during actual chest CT scans.

Among the two-scout views, the lowest dose reduction resulted from using the $90^{\circ}-0^{\circ}$ scout view, while the lowest and highest dose-to-CT-image-quality ratios, 13.32 and 23.64 , resulted from using the $0^{\circ}-90^{\circ}$ and $90^{\circ}-180^{\circ}$ views, respectively. Thus, the $90^{\circ}-0^{\circ}$ scout view resulted in the lowest dose, irrespective of the image quality, whereas the $0^{\circ}-90^{\circ}$ scout view achieved a significant dose reduction while also maintaining high image quality. Our literature review revealed that the dose variations between the $0^{\circ}$ and $180^{\circ}$ scout views were not large enough to significantly affect CT scans in previous studies (7). This behavior was likely observed because the $180^{\circ}$ scout view might lead to doses slightly higher than those resulting from using the $0^{\circ}$ scout view, but it would not reduce the noise level. In the current study, however, both the $0^{\circ}$ and $180^{\circ}$ scout views led to significant $20 \%$ gaps in the CTDI. With the $0^{\circ}$ scout view as a reference, the effective dose also varied from 0.06 $\mathrm{mSv}$ to $0.51 \mathrm{mSv}$, depending on the scout view (Table 2). This radiation dose variation could be considered significant, even the chest CT scan length is relatively short (about 30 $\mathrm{cm})$; in the United States, the effective doses for chest X- 
rays (frontal and lateral chest radiography) and mammography series are generally about $0.065 \mathrm{mSv}$ and $0.42 \mathrm{mSv}$, respectively (4).

In addition, each scout view and angular position at the cavity of the CTDI head phantom yielded a similar result (Figure 6, Table 2). The smallest dose was found in the central cavity of the CTDI head phantom, with increasing doses found in the $180^{\circ}, 90^{\circ}$, and $0^{\circ}$ cavities, for both levels of tube voltage $100 \mathrm{kVp}$ and $120 \mathrm{kVp}$. CTDI is a unit related to the energy of the diagnostic X-ray CT scans performed on the patient. In this study, with increasing $\mathrm{mA}$, the radiation dose was found to be largest in the $0^{\circ}$ cavity of the CTDI head phantom using an ionization chamber, with decreasing values in the $180^{\circ}, 90^{\circ}$, and central cavities (Figure 6). However, at a voltage of $120 \mathrm{kVp}$, there was greater variation in the radiation dose in the central cavity of the CTDI head phantom. Thus, when the tube voltage is lowered from $120 \mathrm{kVp}$ to $100 \mathrm{kVp}$, the radiation dose can be decreased by $53.16 \%$ in the $0^{\circ}$ cavity and by $63.18 \%$ in the central cavity.

In some clinical cases, scans to obtain images are made along only a single axis, rather than both the $\mathrm{x}$ - and $\mathrm{y}$-axes of patients (AP and lateral scout views), during the scout scanning that precedes a helical CT scan. This using only single scout view can lead to inappropriate CT scanning and dose reduction failure using SmartmA. As the frontal scout view alone, without information from lateral scout views, failed to achieve a suitable noise index at the center of the y-axis of the patient in CT scanning, two-scout views are necessary to obtain quality CT images while limiting patient radiation exposure to a proper dose $(9,15)$.

Based on the results of this study, scout angle order could be another determinant of the dose and image quality of CT scanning; therefore it is necessary to select and to apply an optimum scout angle order such as $0^{\circ}-90^{\circ}$. It is also necessary to use the proper radiation dose, and the optimum combination of scout views to achieve "improved CT image quality with the least dose" (ALARA principle) (12, 16).

A limitation of the current study was that only tests conducted in the GE CT unit led to variable results. This variation was most likely due to the properties of the unit that could affect CT studies involving scouts. The CTDI results did not differ significantly between using AutomA alone and using both AutomA and SmartmA, likely because this test was conducted in a single phantom rather than in diverse human body types $(8,9,14)$. In general, AEC adjusts the tube current to the regional body anatomy for the purpose of reducing radiation doses in projection angles. These techniques, along with the information computed from either scout views or in real time, are classified as Smart-scan in GE Healthcare systems, CARE Dose in
Siemens systems, or DOM in Philips systems.

Although available AEC techniques adjust the tube current to obtain identical image quality, the technique (GE Healthcare systems), based on information computed from the scout view, has some limitations. Compared with images obtained in real time, Smart-scan images can vary greatly, depending on the method of obtaining images from the scout view and the information obtained from the scout view. Therefore, adoption of the scout view can vary greatly, effect both image quality and radiation dose.

AEC techniques also increase radiation doses for dense patients and very thin patients. Thus, in these cases, AEC techniques require necessary modifications of $\mathrm{mA}$ ranges to avoid overexposure.

While the majority of studies on the AEC techniques reduce the radiation dose from the tube current modulated in the $\mathrm{x}-, \mathrm{y}-$, and $\mathrm{z}$ planes, based on morphology or radiation attenuation, few studies focus on how the selection of scout views affects the exposure dose and noise values in CT images. We conclude that CT scan users should take into account appropriate managing of the scout views in order to obtain the best CT image quality while reducing the radiation dose and adapting to the noise index of the selected configuration.

\section{Acknowledgments}

None declared.

\section{Footnotes}

Authors' Contributions: Study concepts: Myeong Seong Kim and Jong-Woong Lee; study design: Myeong Seong Kim and Dae Cheol Kweon; definition of intellectual content: Myeong Seong Kim and Dae Cheol Kweon; literature research: Myeong Seong Kim and Jong-Woong Lee; experimental studies: Myeong Seong Kim and Jong-Woong Lee; data acquisition: Myeong Seong Kim and Sun Geun Kim; data analysis: Myeong Seong Kim and Jong-Woong Lee; statistical analysis: Sun Geun Kim and Myeong Seong Kim; manuscript preparation: Myeong Seong Kim and Dae Cheol Kweon; manuscript editing: Jong-Woong Lee and Dae Cheol Kweon; manuscript review: Myeong Seong Kim and Dae Cheol Kweon; each author should have participated sufficiently in the study to take public responsibility for appropriate portions of the content.

Financial Disclosure: None declared.

Funding/Support: The authors declare that they have no conflicts of interest. 


\section{References}

1. Brenner DJ, Hall EJ. Computed tomography an increasing source of radiation exposure. $N$ Engl J Med. 2007;357(22):2277-84. doi: 10.1056/NEJMra072149. [PubMed: 18046031].

2. Lee TY, Chhem RK. Impact of new technologies on dose reduction in CT. Eur J Radiol. 2010;76(1):28-35. doi: 10.1016/j.ejrad.2010.06.036. [PubMed: 20643522].

3. McCollough $\mathrm{CH}$, Primak AN, Braun N, Kofler J, Yu L, Christner J. Strategies for reducing radiation dose in CT. Radiol Clin North Am. 2009;47(1):27-40. doi:10.1016/j.rcl.2008.10.006. [PubMed: 19195532].

4. Smith-Bindman R, Lipson J, Marcus R, Kim KP, Mahesh M, Gould R, et al. Radiation dose associated with common computed tomography examinations and the associated lifetime attributable risk of cancer. Arch Intern Med. 2009;169(22):2078-86. doi: 10.1001/archinternmed.2009.427. [PubMed: 20008690].

5. McCollough $\mathrm{CH}$, Bruesewitz MR, Kofler JJ. CT dose reduction and dose management tools: overview of available options. Radiographics. 2006;26(2):503-12. doi: 10.1148/rg.262055138. [PubMed: 16549613].

6. Lewis MA, Edyvean S. Patient dose reduction in CT. Br J Radiol. 2005;78(934):880-3. doi: 10.1259/bjr/75960844. [PubMed: 16177008].

7. Kalra MK, Rizzo SM, Novelline RA. Reducing radiation dose in emergency computed tomography with automatic exposure control techniques. Emerg Radiol. 2005;11(5):267-74. doi:10.1007/s10140-004-03957. [PubMed: 16133620].

8. Kalra MK, Maher MM, Toth TL, Hamberg LM, Blake MA, Shepard JA, et al. Strategies for CT radiation dose optimization. Radiology. 2004;230(3):619-28. doi: 10.1148/radiol.2303021726. [PubMed: 14739312].
9. Lee $\mathrm{CH}$, Goo JM, Ye HJ, Ye SJ, Park CM, Chun EJ, et al. Radiation dose modulation techniques in the multidetector CT era: from basics to practice. Radiographics. 2008;28(5):1451-9. doi: 10.1148/rg.285075075. [PubMed: 18794318].

10. Leng S, Yu L, McCollough CH. Radiation dose reduction at CT enterography: How low can we go while preserving diagnostic accuracy?.AJR Am J Roentgenol. 2010;195(1):76-7. doi: 10.2214/AJR.10.4676. [PubMed: 20566799].

11. Papadakis AE, Perisinakis K, Damilakis J. Automatic exposure control in pediatric and adult multidetector CT examinations: a phantom study on dose reduction and image quality. Med Phys. 2008;35(10):4567-76. doi: 10.1118/1.2977535. [PubMed: 18975703].

12. ICRP. . ICRP Publication 87: Managing patient dose in computed tomography. ; 2010.

13. Kalender WA, Wolf $\mathrm{H}$, Suess $\mathrm{C}$. Dose reduction in $\mathrm{CT}$ by anatomically adapted tube current modulation. II. Phantom measurements. Med Phys. 1999;26(11):2248-53. doi:10.1118/1.598738. [PubMed:10587205].

14. Althen JN. Automatic tube-current modulation in CT-a comparison between different solutions. Radiat Prot Dosimetry. 2005;114(1-3):30812. doi: 10.1093/rpd/nch501. [PubMed: 15933127].

15. Samelson EJ, Christiansen BA, Demissie S, Broe KE, Zhou Y, Meng CA, et al. Reliability of vertebral fracture assessment using multidetector CT lateral scout views: the Framingham Osteoporosis Study. Osteoporos Int. 2011;22(4):1123-31. doi: 10.1007/s00198-010-1290-6. [PubMed: 20495902].

16. Mayo JR, Aldrich J, Muller NL, Fleischner S. Radiation exposure at chest CT: a statement of the Fleischner Society. Radiology. 2003;228(1):15-21. doi: 10.1148/radiol.2281020874. [PubMed: 12832569]. 